\title{
THE CLINICAL IMPORTANCE OF PD-L1 AND PD-1 EXPRESSION IN DIFFUSE LARGE B CELL LYMPHOMA
}

\section{Adelina BIRCEANU COROBEA ${ }^{1}$, Anca EVSEI ${ }^{1}$, Cristian ROSIANU ${ }^{2}$, Mircea GHEORGHE ${ }^{3,4} \bowtie$, Gabriela BIRCEANU ${ }^{1}$, Maria SAJIN ${ }^{5}$, Anca LUPU ${ }^{6}$, Narcis COPCA ${ }^{7}$}

${ }^{1}$ Department of Pathology, „Sfanta Maria“ Clinical Hospital, Bucharest, Romania

2 Department of Gastroenterology „Sfanta Maria“ Clinical Hospital, Bucharest, Romania

${ }^{3}$ Department of General and Esophageal Surgery, „Sfanta Maria“ Clinical Hospital, Bucharest, Romania

${ }^{4}$ Department of Surgery, „Carol Davila“ University of Medicine and Pharmacy, Bucharest, Romania

${ }^{5}$ Department of Pathology, „Carol Davila“ University of Medicine and Pharmacy, Emergency University Hospital, Bucharest, Romania

${ }^{6}$ Department of Hematology, „Carol Davila“ University of Medicine and Pharmacy, „Coltea“ Clinical Hospital, Bucharest, Romania

${ }^{7}$ Department of Surgery,"Sfanta Maria“ Clinical Hospital, Bucharest, Romania

Received 03 July 2018, Accepted 14 Aug 2018

https://doi.org/10.31688/ABMU.2018.53.3.01

\section{Abstract}

Introduction The target pathway programmed cell death-1 (PD-1)/ programmed death-ligand 1 (PD-L1) shows clinical efficacy in solid tumors, but also in Hodgkin's and non-Hodgkin's lymphoma. PD-L1 expression most often correlates with a poor prognosis and a PD-1 regulatory factor that mediates immunosuppression. The presence of an increased number of tumor-infiltrating lymphocytes (TILs) PD-1 + is a favorable prognostic factor in patients with diffuse large B cell lymphomas (DLBCLs) and follicular lymphomas, while the low number of TILs PD-1 + is associated with an increased risk of histological transformation. In DLBCLs, TILs PD-1 + may not reflect the depletion of T-mediated tumor cells but may be an indicator of lymphoid cell origin.

Objectives The objective of this study was to describe the correlation between PD-1 and PDL-1 with

\section{Résumé}

L'importance clinique de l'expression de PD-L1 et PD-1 dans le lymphome diffus à grandes cellules B

Introduction La voie cible PD-1 / PD-L1 présente une efficacité clinique dans les tumeurs solides mais également dans les lymphomes de Hodgkin et non-Hodgkin. L'expression de PD-L1 est le plus souvent corrélée à un mauvais pronostic et à un facteur régulateur de PD-1 qui induit une immunosuppression. La présence d'un nombre accru de lymphocytes infiltrant la tumeur (TIL) PD-1 + est un facteur pronostique favorable chez les patients atteints de DLBCL et de lymphomes folliculaires, tandis que le faible nombre de TIL PD-1 + est associé à un risque accru de transformation histologique. Dans DLBCL, TIL PD-1 + peut ne pas refléter l'épuisement des cellules tumorales à médiation $\mathrm{T}$ mais peut être un indicateur de l'origine des cellules lymphoïdes. 
survival in patients with the diagnosis of diffuse large B cell lymphoma.

Material and methods We have studied 80 patients and we have analyzed DLBCLs according to the Hans algorithm; in addition, we analyzed PD-1 and PD-L1 in tumor cells and in immune cells and we correlated this data with patient's survival.

Results We found that there is a tendency of decreased survival and therapeutic response in DLBCL patients, with both an intense and weak PD-L1 positivity in tumor cells. PD-1 low positivity was associated in higher percentage with relapse and treatment unresponsiveness.

Conclusions Our data suggested that PD-L1 expression correlates with a poor clinical response although it is not an independent prognostic marker and PD-1 represents a favorable prediction factor for survival.

Keywords: diffuse large B cell lymphomas, PD-L1, PD-1, prognostic, survival, therapeutic response.

\section{INTRODUCTION}

Diffuse large B-cell lymphoma (DLBCL) is the most common (exceeds 35\%) of non-Hodgkin's malignant lymphomas (NHL). It represents a problem of national and international health because it occurs at all ages, without specific signs and symptoms, has an aggressive pattern of evolution and a poor prognosis if left untreated. The treatment for DLBCL patients has been revolutionized in recent years, with the addition of rituximab to combination chemotherapy, resulting in an increased proportion of cured patients.

DLBCL is characterized by a high heterogeneity in morphology, immunophenotype, and cytogenetic profile, some clinical aspects such as the type of treatment response and evolution. DLBCL is classified by gene expression profile (GEP) into germinal center cell-like (GCB) group, activated B cell-like (ABC) group and type 3 , with the last two having significantly worse outcome ${ }^{1}$. Hans algorithm is made up of three markers (CD 10: CGB, BCL 6 associated with both CGB and ABC group; MUM1: post CG marker) $)^{2}$.

The tumor microenvironment is the cellular and molecular environment which coexists with tumor cells and continuously interacts with them. Recognition of the microenvironment importance, especially in B malignancies, paved the way for the development of targeted therapeutic strategies as well as for the interaction with tumor cells.
Objectifs Les objectifs de l'étude sont les corrélations entre PD-1 et PDL-1 avec la survie.

Matériel et méthodes Nous avons étudié 80 patients et nous avons analysé DLBCL selon l'algorithme de Hans; de plus, nous avons analysé PD-1 et PD-L1 dans les cellules tumorales et dans les cellules immunitaires et nous avons corrélé ces données avec la survie du patient.

Résultats Nous avons constaté une tendance à la diminution de la survie et de la réponse thérapeutique chez les patients atteints de DLBCL, avec une positivité à la fois intense et faible au PD-L1 dans les cellules tumorales. La faible positivité PD-1 était associée à un pourcentage plus élevé de rechute et de manque de réponse au traitement.

Conclusions Nos données suggèrent que l'expression de PD-L1 est en corrélation avec une réponse clinique médiocre, bien que ce ne soit pas un marqueur pronostique indépendant et que PD-1 représente un facteur de prédiction favorable pour la survie.

Mots-clés: lymphome diffus à grandes cellules B, PD-L1, PD-1, pronostic, survie, réponse thérapeutique.

Many studies have evaluated the role of PD-1 (programmed cell death-1) and PD-L1 (programmed death-ligand 1), which is expressed in most aggressive B lymphomas but also correlates with immune escape mechanisms $\mathrm{s}^{3.5}$.

The MHC II transactivator class (CIITA) fuses frequently with PD-L1 and PD-L2 that may result from decreased HLA-DR expression ${ }^{3}$. The pathway consisting of the PD-1 receptor (CD 279) and its PD-L1 and PD-L2 ligands (B7-DC; CD 273) plays an essential role in peripheral tolerance and also mediates the inhibitory signals constituting antitumor immunity. The target pathway PD-1/ PD-L1 shows clinical efficacy in solid tumors ${ }^{6}$ but also in Hodgkin's and non-Hodgkin's lymphoma ${ }^{7,8}$.

Kiyasu et al evaluated the PD-L1 expression in 1253 patients diagnosed with DLBCL; the results of their study reveal the significance of identifying PD-L1 expression that most often correlates with a poor prognosis and a PD-1 regulatory factor that mediates immunosuppression. The tumor infiltrating lymphocytes (TIL) PD-1 positive number correlates with tumor-specific PD-L1 expression and represents a negative prognostic factor. The PD-L1 expression (PD-L1 or mouse PD-L1 positive) is associated with the non-germinal subtype (CGB) and the presence of EBV. The number of PD-1 positive in TIL decreases proportionally with PD-L1 expression in malignant or non-malignant cells compared to DLBCL CGB, where low PD-L1 expression is observed. The low 
number of PD-1 TIL correlates with the presence of B-symptoms, extra lymph node localization, bulky tumor masses and with lower survival compared to negative PD-L1 patients. Death in lymphoid pathology correlated with disease progression and is more common in PD-L1 positive patients, but there is no significant difference between mPD-L1 positive and mPD-L1 negative?.

The presence of an increased number of TILs PD-1 positive is a favorable prognostic factor in patients with DLBCL and follicular lymphomas, while the low number of TILs PD-1 positive is associated with an increased risk of histological transformation $^{8,9}$.

In DLBCL, TILs PD-1 positive may not reflect the depletion of T-mediated tumor cells but may be an indicator of lymphoid cell origin. PD-1 is highly expressed in the germinal center level in the $\mathrm{T}$ helper lymphocytes ${ }^{10}$.

The increased TILs PD1 count is associated with a good prognosis and these aspects are not influenced by the presence of PD-L1 in DLBCL or non-malignant cells in the tumor microenvironment. Patients with a low PD-1 positive TIL and PD-L1 expression are associated with a worse prognosis compared to the group of patients who have PDL1 or m PD-L1 negativity. DLBCL PD-L1 positive shows a low PD-1 TIL. Identifying PD-L1-specific cell expression in DLBCL may have prognostic significance, but may also represent a therapeutic decision ${ }^{11}$.

Promising treatments for B-cell malignancies are targeted therapies for $\mathrm{B}$ cell receptor (BCR) signaling, PD-1 (programmed death-1) and T-cell chimerical T-cell receptors (CAR-T- cell).

However, the contribution of the tumor microenvironment to diffuse large B cell lymphoma, pathogenesis and tumor survival is not fully understood. The main objective of understanding tumor biology as well as tumor microenvironment is the improvement of diagnosis, classification, prognosis and development of new therapeutic strategies in hematological malignancies.

The objective of the study was to describe the correlation between PD-1 and PD-L1 with survival in patients with diffuse large $B$ cell lymphoma

\section{Material AND MEthods}

We conducted a retrospective study carried out over a period of 5 years, including 80 patients treated in the Hematology Department of Coltea Clinical Hospital Bucharest, Romania. The histopathological and immunohistochemical stains were performed in the Department of Pathology of Coltea Clinic
Hospital and The University Emergency Hospital Bucharest, Romania.

Patient material was anonymized; the study was approved by the Ethical Committee of Coltea Clinical Hospital Bucharest and was performed according to the Declaration of Helsinki standards. The mean follow up was 72 months. The inclusion criteria were: diagnosis of diffuse large B cell lymphoma and were collected according to their morphology and confirmed with immunohistochemical stains according to the WHO 2016 classification for the mature lymphoid B cell tumors. We also examined different immunohistochemical features including PD-1 and PD-L1.

The following antibodies and staining conditions were used: CD 20 (Novocastra at a dilution of 1:100), CD 10 (Novocastra at a dilution of 1:60), MUM 1 (Novocastra at a dilution of 1:100), BCL 6 (Novocastra at a dilution of 1:50), PD-L1 (Biocare Medical CAL 10 at a dilution of 1:75), PD-1 (Cell Marque 1:75).

For statistical analysis, staining results were categorized by immunoreactivity scores (IRS) for all the antibodies staining intensity and percentage of positive tumor cells were scored according to Remmele score 9 . Percentage of stained tumor cells were categorized and had percentages as follows: CD 10 and BCL6 were positive when they are positive over $30 \%$, MUM1 over 40\%, PD-1 was high when there were more than 30 cells/ $10 \mathrm{HPF}$ and low when there were under 30 cells/ 10 HPF (Fig. 1, Fig. 2). For PD-L1 quantification, it was considered positive when cells had a membrane and/ or cytoplasm positivity less than $10 \%$. These scores values were then multiplied by points given for staining intensity ( 0 was negative, 1 weak staining and 2 intense staining) (Fig. 3, Fig. 4).

For the univariate Cox analyses, the Likelihood Ratio or Fisher and Mann-Whitney were used to compare continuous and discontinuous variables. Survival analysis was conducted with the Kaplan-Meier method compared with the Log-rank test. The p-value < 0.05 was interpreted as statistically significant. The analyses were carried out with $\mathrm{R}$ statistical software.

\section{Results}

\section{Patient's selection and clinical data}

For the total cohort of 80 patients, we examined the morphological features and we also had established gene expression profile (GEP) with immunohistochemical stains.

The samples were collected from 41 male patients (51.2\%) and 39 female patients (48.8\%). The median age was 57 years with a minimum of 19 years and a maximum of 87 years. 


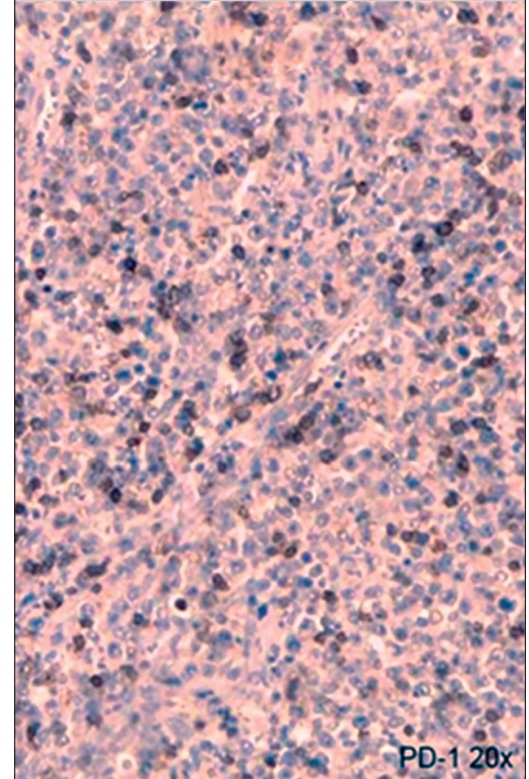

Fig. 1. PD-1 20x positive in more than 30 cells/10HPF in microenvironment lymphocytes.

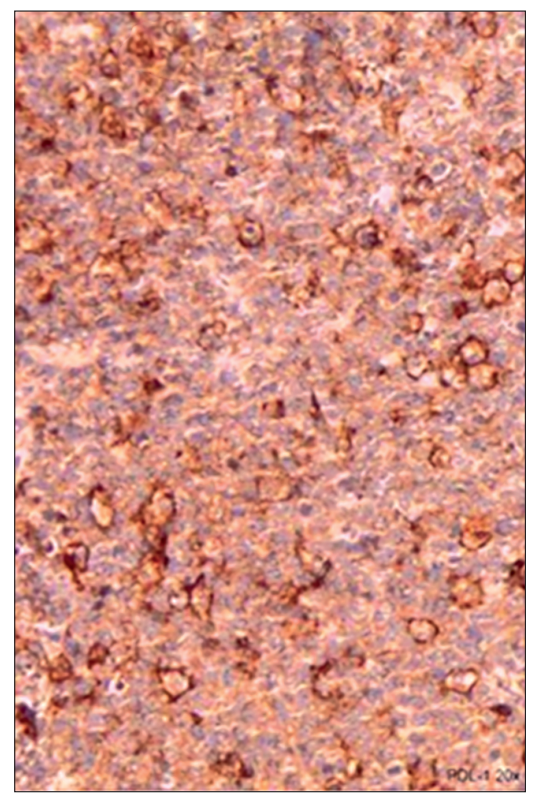

Fig. 3. PDL-1 20x tumor cells positive in more than $10 \%$.

The B symptoms presence (weight loss, profuse sweats, and fever without infectious causes) is considered an unfavorable prognostic factor. 44 patients from the cohort $(55 \%)$ had B symptoms at the diagnosis time; the remaining 36 patients (45\%) were asymptomatic. According to the Ann Arbor classification, most patients (63.3\%) came in with an advanced stage (stage III and IV), the majority being in stage IV.

More than a half of the patients in the cohort $(55.7 \%)$ presented at the onset International Prognostic Index (IPI) 2 index on the second place being followed by the IPI index 3 in a percentage of

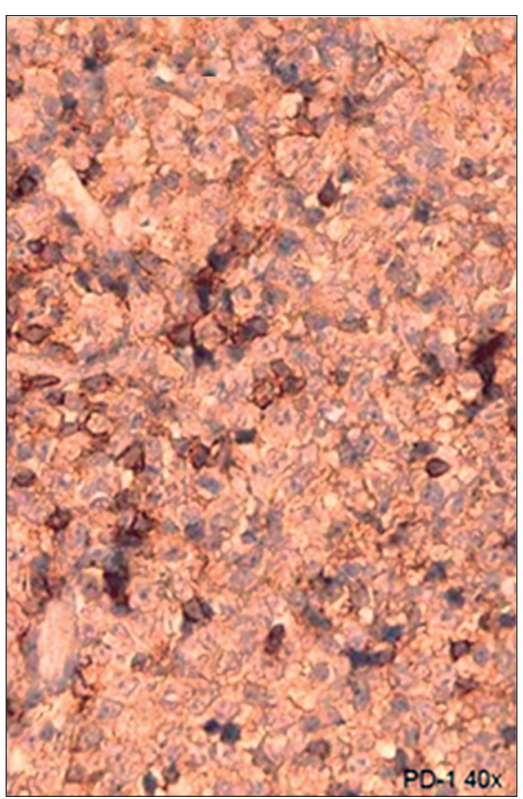

Fig. 2. PD-1 40x positive in less than 30 cells/10HPF in microenvironment lymphocytes

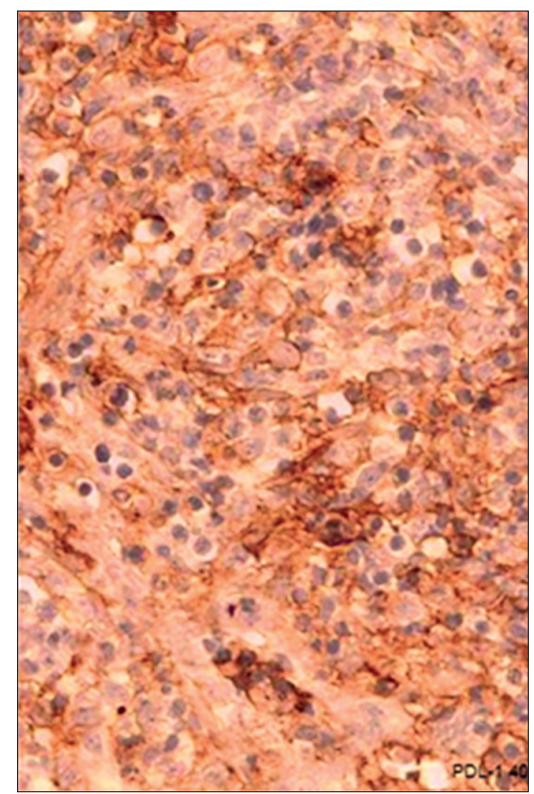

Fig. 4. PDL-1 40x tumor cells positive in more than $10 \%$.

$27.8 \%$, IPI I (8.9\%) and IPI 4 (5.1\%), and in the last, the IPI index 5 in the proportion of $2.5 \%$.

Clinical performance status (ECOG) - approximately 3 quarters $(63.8 \%)$ of patients presented in ECOG 2, while ECOG 3 was represented in $33.8 \%$ of cases, the remaining $2.5 \%$ being represented by ECOG 4. Bulky disease was present in 25 patients $(31.3 \%)$ of total patients in the studied group, the remaining $55(68.8 \%)$ did not show bulky disease.

According to the Hans algorithm, the classification of high-grade diffuse B cell lymphoma was 
represented by 33 patients diagnosed with germinal center (CGB) and 39 with germinal activated cell/ non-center (non- CGB). The complete therapeutic response was recorded in 47 patients, representing slightly over half (58.8\%), while 24 had a partially therapeutic response, the remaining $11.3 \%$ were non-responsive. In $30 \%$ of cases (24 patients) relapse was recorded and in approximately three quarters and 56 patients, respectively, did not relapse.

Regarding overall survival (OS), in the male cohort, only one death was registered while 3 deaths were registered in the female cohort. The average estimated survival time to death was 103.52 months for women, and 111.50 months for men with a $95 \%$ confidence index. The median survival time was calculated only for men and was 103 months.

\section{Comparison of immunohistochemical staining for PD-L1 and PD-1}

Patients who presented increased PD-1 were associated in $100 \%$ and $96.6 \%$ with PD-L1 negative and respectively weak positive.

CGB was associated in increased proportion $(60 \%)$ with the negativity of PD-L1 in tumor cells and $86.2 \%$, respectively $10.9 \%$ with weak and intense PD-L1 positivity in tumor cells. Non-CGB type was associated in $89.1 \%$, respectively $13.8 \%$ with the PD-L1 intense and poor tumor cells positivity and in less than $40 \%$ with the negativity of PD-L1.
The gene profile germinal center type was associated in $84.4 \%$ with increased immune cells PD-1 positivity, while immune cells with low PD-1 was associated in less than $10.6 \%$ with CGB. DLBCL non-CGB type was associated in $89.4 \%$ with PD-1 low positivity and in $15.6 \%$ with immune cells increased positivity. As it is apparent from the analysis of the studied cohort, another significant statistical association was between clinical parameters and immunohistochemical stains as follows: the absence of B symptoms correlates with the PD-1 low positivity in immune cells, while patients who presented B symptoms had increased PD-1 expression in the immune cells.

The evaluation of PD-L1 in our study showed the following: most patients (46), presented PD-L1 positivity in tumor cells, while 29 of them showed a PD-L1 weak positivity in tumor cells, with an equal number of deaths between these variables.

Analyzing the survival curves we found that there is a tendency of decreased survival in patients with an intense and weak PD-L1 positivity in tumor cells (Table 1).

Regarding the tumor microenvironment of PD-1 in TIL, 47 patients of the study group presented low positivity and 2 of them succumbed. The remaining 32 patients showed increased positivity PD-1 in microenvironment $\mathrm{T}$ lymphocytes. The average survival until death for the increased PD-1 in lymphocytes from

Table 1. Survival curves according to PDL-1 expression

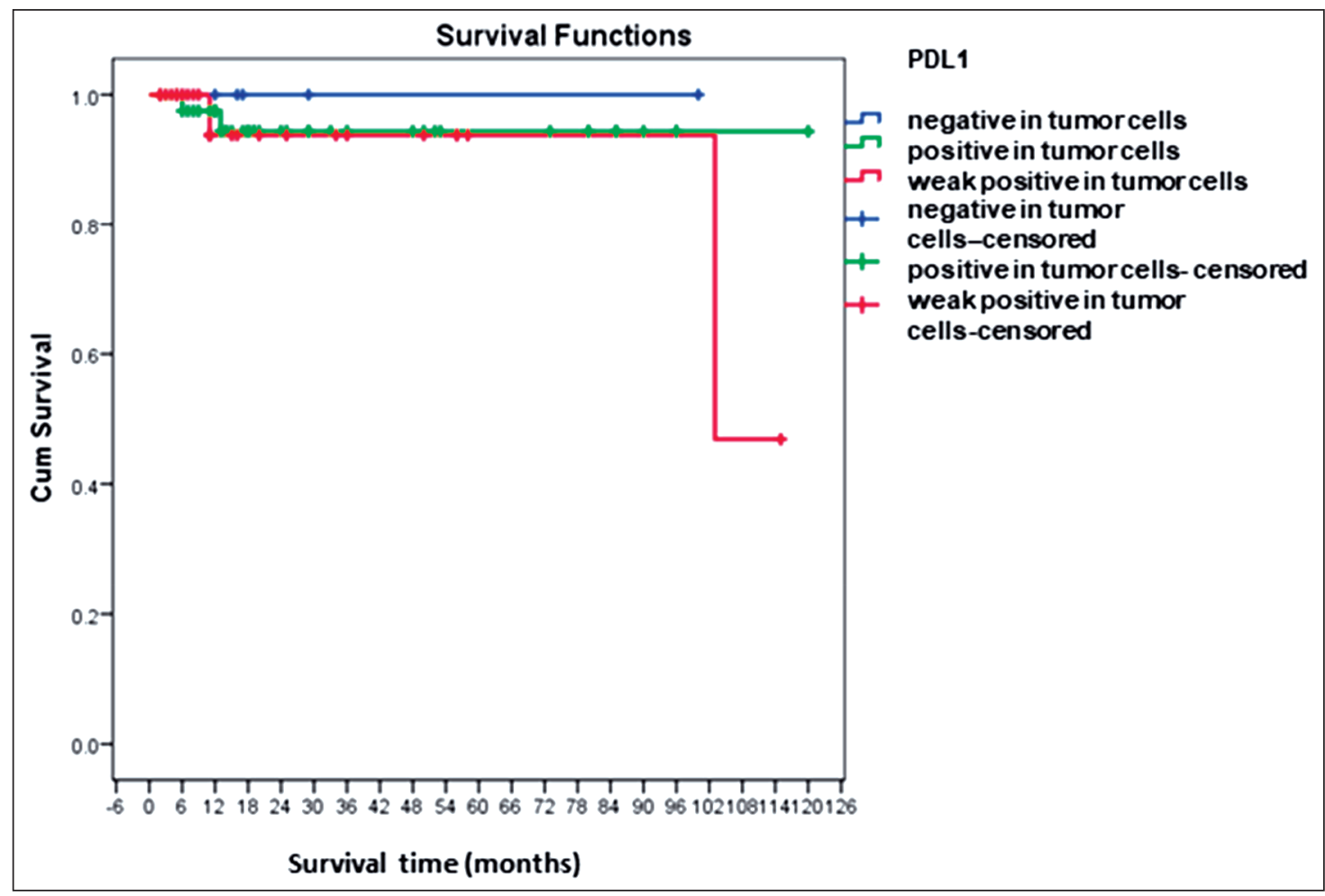


the tumor microenvironment patients was 104,10 months with a standard deviation of 6,249 months, while the median survival was 103,00 months (Table 2, Table 3).

PD-1 high positivity in immune cells was associated in $43.5 \%$ (20 patients) of cases with complete therapeutic response and in $16.75 \%$ (1 patient) of cases were non-responsive, since the patients with low positivity, associated in $56.5 \%$ (26 patients) of cases complete therapeutic response and $83.3 \%$ (5 patients) were non-responsive.

Regarding therapeutic response in patients with negative PD-L1 in tumor cells, $6.4 \%$ presented a complete therapeutic response and only $4.2 \%$ partially therapeutic response and any of them was non-responsive.

Most patients who had PD-L1 high positivity in tumor cells, (83.3\%) were non-responsive, $62.5 \%$ had a partial therapeutic response and fewer than half $(53.2 \%)$ presented with a complete therapeutic response.

The weak positivity of PD-L1 in tumor cells was associated in a high percentage $(40.4 \%)$ with a full therapeutic response, $33.3 \%$ with a partial therapeutic response and the fewest (16.7\%) were non-responsive.

The relapse had been registered in CGB patients in $27.3 \%$ while non-CGB experienced relapse in $31.9 \%$ of cases.

Patients who had intense and low tumor cells PD-L1 positivity relapsed in $32.6 \%$ and $24.1 \%$, respectively, while patients who had tumor cells PD-L1 negativity had presented relapse in $40 \%$ of cases. Patients who presented an increased PD-1 percentage

Table 2. Survival according to PD-1 expression Case Processing Summary

\begin{tabular}{ccccc}
\hline \multirow{2}{*}{ PD 1 } & \multirow{2}{*}{ Total N } & \multirow{2}{*}{ N of Events } & \multicolumn{2}{c}{ Censored } \\
\cline { 4 - 5 } & & & $N$ & Percent \\
\hline High & 32 & 2 & 30 & $93.8 \%$ \\
\hline Low & 47 & 2 & 45 & $95.7 \%$ \\
\hline Overall & 79 & 4 & 75 & $94.9 \%$ \\
\hline
\end{tabular}

in immune cells had associated in $28.1 \%$ of cases with relapse, while patients with low tumor microenvironment PD-1 had associated in $31.9 \%$ of cases with relapse.

\section{Discussion}

In the study cohort, men were dominant (51.2\%), with men/women ratio of $1.05 / 1$, similar to literature data, in which a mild predominance of men compared to women is mentioned ${ }^{12}$. The patients' age analysis at the time of diagnosis showed a negative distribution, indicating more than $50 \%$ of patients older than the median age (57 years). Compared to the literature, in which most patients are diagnosed in the $7^{\text {th }}$ or $8^{\text {th }}$ decade, we found a mean age of 57 years in our study. The literature data demonstrate that the age at diagnosis can predict the clinical outcome $e^{13-15}$.

The B signs were positive in $55 \%$ of patients, compared with data from the literature in which a percentage of $40 \%$ of patients had B symptoms reported. The presence of B symptoms, according to the literature, is an independent negative prognostic factor for overall survival, predisposing to the failure of the therapy, decreasing the chances of a complete response. Analyzing the survival curves depending on the disease stage, a statistically significant decrease is observed for the advanced disease group, with 4 death events recorded during the first 18 months and increased frequency of relapse.

Prognostic factors for patients with aggressive lymphomas are very important because they are necessary for selecting patients who have increased recurrence risk, being candidates from the beginning to a more intensive therapy ${ }^{16-19}$. Analyzing survival data, there were 3 deaths and a decrease in survival in the first 6 months of diagnosis in patients enrolled in the activated cell subtype and only one death in DLBCL-type germline patients. Literature studies have shown that patients with DLBCL CGB type have a better prognosis after the introduction of Rituximab.

Table 3. Overall survival according to PD-1 expression

\begin{tabular}{|c|c|c|c|c|c|c|c|c|}
\hline \multicolumn{9}{|c|}{ Means and Medians for Survival Time } \\
\hline \multirow{3}{*}{ PD 1} & \multicolumn{4}{|c|}{ Mean $^{a}$} & \multicolumn{4}{|c|}{ Median } \\
\hline & \multirow{2}{*}{ Estimate } & \multirow{2}{*}{ Std. Error } & \multicolumn{2}{|c|}{ 95\% Confidence Interval } & \multirow{2}{*}{ Estimate } & \multirow{2}{*}{ Std. Error } & \multicolumn{2}{|c|}{ 95\% Confidence Interval } \\
\hline & & & Lower Bound & Upper Bound & & & Lower Bound & Upper Bound \\
\hline High & 104.100 & 6.249 & 91.851 & 116.349 & 103.000 & . & . & . \\
\hline Low & 113.785 & 4.290 & 105.376 & 122.194 & . & . & . & . \\
\hline Overall & 108.687 & 5.416 & 98.072 & 119.303 & . & . & . & . \\
\hline
\end{tabular}


Tumor cells or immune cells PD-1 and PD-L1 expression is a valuable parameter in the prediction of PD-1/ PD-L1 immunotherapy responsiveness. In our case, PD-1 has been extensively expressed in immune cells and PD-L1 has been extensively expressed in tumor cells suggesting that the PD-1/ PD-L1 pathway may be useful in some patients with DLBCL. According to the literature studies, Dohee concluded that increased expression of PD-1 reflects an active immune response, with favorable survival rate ${ }^{20-23}$.

Cox analysis has demonstrated PD-1 as a prognostic marker favoring overall survival (OS). Kyasu et al suggested that PD-L1 expression correlates with a poor clinical response although it is not an independent prognostic marker, suggesting that target treatment with PD-L1 might be beneficial for some patients with DLBCL. PD-1 is a favorable survival prediction factor and is an independent DLBCL prognostic marker, and PD-L1 is associated with poor $\operatorname{prognosis}^{10}$

\section{Conclusions}

The present study suggests that PD-1 or PD-L1 could be potential biomarkers for targeted treatment in some patients diagnosed with DLBCL. Also, we could prove an association between an increased percentage of PD-L1 intense positive with the non-CGB type which, once again, gives an unfavorable prognosis; the CGB profile has been associated with a high percentage of elevated PD-1 in immune cells, which gives the latter a favorable prognosis, according to literature data.

\section{Acknowledgements:}

All the authors contributed equally to the presented study and take responsibility for the integrity of the data and the accuracy of the data analysis.

\section{Compliance with Ethics Requirements:}

„The authors declare no conflict of interest regarding this article"

"The authors declare that all the procedures and experiments of this study respect the ethical standards in the Helsinki Declaration of 1975, as revised in 2008(5), as well as the national law. Informed consent was obtained from all the patients included in the study"

"No funding for this study"

\section{References}

1. Coutinho R, Clear AJ, Owen A, Wilson A, et al. Poor concordance among nine immunohistochemistry classifiers of cell-of-origin for diffuse large B-cell lymphoma: implications for therapeutic strategies. Clin Cancer Res 2013; 19:66866695.

2. Hans CP, Weisenburger DD, Greiner TC, et al. Confirmation of the molecular classification of diffuse large B-cell lymphoma by immunohistochemistry using a tissue microarray. Blood 2004;103:275-282.

3. Brusa D, Sara Serra, Marta Coscia, et al. The PD-1/PD-L1 axis contributes to T-cell dysfunction in chronic lymphocytic leukemia. Haematologica 2013; 98(6):953-963.

4. Steidl C, Shah SP, Woolcock BW, et al. MHC class II trans activator CIITA is a recurrent gene fusion partner in lymphoid cancers. Nature 2011; 471(7338):377-381.

5. Chen BJ, Chapuy B, Ouyang J, et al. PD-L1 expression is characteristic of a subset of aggressive B-cell lymphomas and virus-associated malignancies. Clin Cancer Res 2013;19 (13):3462-3473.

6. Topalian SL, Hodi FS, Brahmer JR, et al. Safety, activity, and immune correlates of anti-PD-1 antibody in cancer. $N$ Engl J Med 2012;366(26):2443-2454.

7. Bachy E, Coiffier B. Anti-PD1 antibody: a new approach to treatment of lymphomas. Lancet Oncol 2014; 15(1):7-8.

8. Ansell SM, Lesokhin AM, Borrello I, et al. PD-1 blockade with nivolumab in relapsed or refractory Hodgkin's lymphoma. N Engl J Med 2015; 372 (4): 311-319.

9. Kim JR, Moon YJ, Kwon KS, et al. Tumor infiltrating PD1-positive lymphocytes and the expression of PD-L1 predict poor prognosis of soft tissue sarcomas. PLoS One 2013; 8(12):82870.

10. Kiyasu J, Miyoshi H, Hirata A, et al. Expression of programmed cell death ligand 1 is associated with poor overall survival in patients with diffuse large B-cell lymphoma. Blood 2015;126 (19): 2193-2201

11. Remmele W, Stegner HE. Recommendation for uniform definition of an immunoreactive score (IRS) for immunohistochemical estrogen receptor detection (ER-ICA) in breast cancer tissue. Pathologe 1987;8(3):138-140.

12. Bernstein L, Petersen B, Mascarenhas J. Epidemiology of hairy cell leukemia in Los Angeles County. Cancer Res 1990; 50: 3605-3609.

13. Flowers CR, Shenoy PJ, Borate U, et al. Examining racial differences in diffuse large B-cell lymphoma presentation and survival. Leuk Lymphoma 2013;54 (2): 268-76.

14. Minard-Colin V, Brugières L, Reiter A, et al. Non-Hodgkin lymphoma in children and adolescents: progress through effective collaboration, current knowledge, and challenges ahead. J Clin Oncol 2015; 33: 2963-2974.

15. Alexander DD, Mink PJ, Adami HO, et al. Multiple myeloma: a review of the epidemiologic literature. Int $J$ Cancer 2007; 120: 40-61.

16. Diaconu C, Arsene D, Balaceanu A, Bartos D. A rare tumor revealed by abdominal trauma: case presentation. Romanian Journal of Morphology and Embryology 2014;55(3):973-976.

17. Dumitras IC, Ionescu C, Bartos D, Diaconu C. The diagnosis of malignant disease: sometimes a matter of pure chance. Arch Balk Med Union 2017;52(1):112-116.

18. Paraschiv B, Dediu G, Iancu A, Bratu O, Diaconu C. Superior vena cava syndrome - review. Arch Balk Med Union 2017;52(1):39-43 
19. Marcu RD, Spinu DA, Socea B, et al. Castleman's disease clinical, histological and therapeutic features. Rev Chim 2018;69(4):823-830.

20. Cerhan JR, Berndt SI, Vijai J, et al. Genome-wide association study identifies multiple susceptibility loci for diffuse large B cell lymphoma. Nat Genet 2014; 46: 1233-1238.

21. Hallack Neto AE, Teruya-Feldstein J, Puig P, et al. P63 protein expression in high risk diffuse large B-cell lymphoma. J Clin Pathol 2009; 62:77-9
22. Taube JM, Klein A, Brahmer JR, et al. Association of PD-1, PD-1 ligands, and other features of the tumor immune microenvironment with response to anti-PD-1 therapy. Clin Cancer Res 2014;20:5064-74.

23. Herbst RS, Soria JC, Kowanetz M, et al. Predictive correlates of response to the anti-PD-L1 antibody MPDL3280A in cancer patients. Nature 2014; 515:563-7. 